\title{
Analytical solution of the simple nonlinear radiative transfer problem
}

\author{
H. V. Pikichyan ${ }^{1 *}$ \\ ${ }^{1}$ NAS RA V. Ambartsumian Byurakan Astrophysical Observatory (BAO), Armenia \\ *E-mail: hovpik@gmail.com,hovpik@bao.sci.am
}

\begin{abstract}
Exploring the "Principle of invariance" and the method of "Linear images", the simple nonlinear conservative problem of radiative transfer is analyzed. The solutions of nonlinear reflection-transmission and internal field problems of one dimensional scattering-absorbing medium of finite optical thickness are obtained, whereas both boundaries of medium illuminated by powerful radiation beams. Using two different approaches - a direct and inverse problem, the analytical solution of the internal field problem is derived.
\end{abstract}

\section{Introduction}

The properties of the scattering-absorbing medium in the linear theory of radiation transfer are assumed to be known in advance. While by solving the radiative transfer equation, the random walk properties of radiation can be determined from the analysis of the multiple interactions between the radiation and matter. But in nonlinear theory of radiative transfer, due to multiple interactions between the radiation and matter (Ambartsumian 1964a), the initial optical properties of both the radiation and absorbingscattering medium are mutually affecting each other, in self-consistent manner. Indeed, if the intensity of an exciting radiation is higher, then some statistically measured fraction of atoms in medium is permanently resided in excited state, i.e. they do not absorb photons. This consequently decreases the value of absorption coefficient of each elementary volume of the medium, until the latter becomes more transparent (Ambartsumian 1964b). In nonlinear case, therefore, it is necessary to take into account the dependence of optical parameters of medium from the intensity of external exciting radiation. The Fig. 1 schematically plots the photon diffusion processes in linear (right) and nonlinear (left) cases. The unshaded circles denote the atoms being in the excited state, which are already eliminated from the multiple absorbing-scattering processes of the medium. 

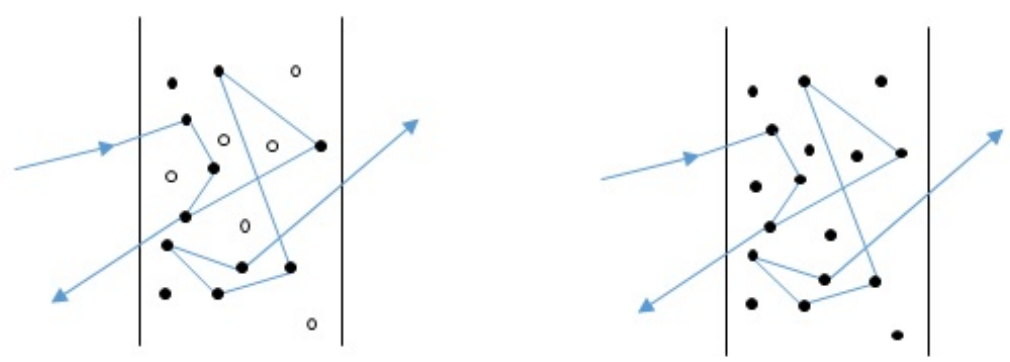

Figure 1: The photon diffusion processes in linear (right) and nonlinear (left) cases.

A goal of this report is to show on the example of simple one-dimensional conservative and isotopically scattering medium of two-level atoms: a) the affection of the mentioned above a non-linearity feature (including also the induced radiation) on properties of the solution of reflection-transmission problem, and on the internal field intensity in medium of a finite optical thickness; b) an efficiency of the implications previously introduced concept of "Linear Images (LI)" (Pikichyan 2016, 2014) together with Ambartsumian's principle of invariance (Ambartsumian 1964a, Pikichyan 2010) for analyzing nonlinear problems of radiative transfer theory.

\section{Two properties of "inverse" and "direct" formu- lation of the same internal field problem}

Let us to consider the one-dimensional, conservatively and isotropically absorbing-scattering medium of a finite optical thickness, consisted of twolevel atoms (Fig. 2). The medium is continuously illuminated from its both boundaries by powerful beams of exciting radiation of the given intensities $x$ (left) and $y$ (right). It is required to find internal $I^{ \pm}$and outgoing $u, v$ intensities of diffuse radiation field.

We may invoke the Boltzmann kinetic equation, written for the "photon gas" (the radiative transfer equation), usually formulating the "twopoint" boundary value problem for determining the radiation intensity $I^{ \pm} \equiv$ $I^{ \pm}\left(\tau, x, y, \tau_{0}\right)$, at the optical depth $\tau$, for the medium of finite optical thickness $\tau_{0}\left(\tau \in\left[0, \tau_{0}\right]\right)$ :

$$
\left\{\begin{array}{c}
\frac{d I^{ \pm}}{d \tau}= \pm \alpha^{ \pm}\left(I^{+}, I^{-}\right) \\
\left.I^{+}\right|_{\tau=0}=x,\left.\quad I^{-}\right|_{\tau=\tau_{0}}=y
\end{array}\right.
$$




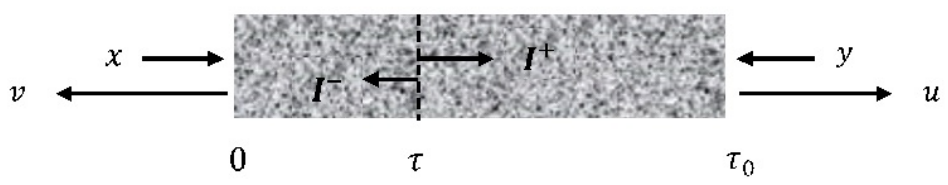

Figure 2: The one-dimensional isotropic, conservatively and isotropically absorbing-scattering medium of finite optical thickness.

where

$$
\alpha^{ \pm}\left(I^{+}, I^{-}\right)=\mp \frac{I^{+}-I^{-}}{2\left[1+b \cdot\left(I^{+}+I^{-}\right)\right]}
$$

is the known specified form of "integral of collisions" and

$$
\begin{aligned}
d \tau=k_{0}(l) d l, \quad l & \in\left[0, l_{0}\right], \quad k_{0}(l)=n(l) \frac{h \cdot \nu}{2} B_{12}, \\
b & \equiv \frac{B_{12}+B_{21}}{2 A_{21}}=\frac{c^{2}}{4 h \nu^{3}}\left(1+\frac{g_{2}}{g_{1}}\right) .
\end{aligned}
$$

The $l$ and $l_{0}$ are geometrical parameters of one-dimensional medium, respectively, depth and thickness. Also, the micro quantities are given in conventional notations (Ivanov 1969).

The Cauchy problem for the same radiative transfer equation can be formulated as an "inverse" one:

$$
\left\{\begin{array}{c}
\frac{d I^{ \pm}}{d \tau}= \pm \alpha^{ \pm}\left(I^{+}, I^{-}\right) \\
\left.I^{+}\right|_{\tau=0}=x,\left.\quad I^{-}\right|_{\tau=0}=v, \quad \text { or }\left.\quad I^{+}\right|_{\tau=\tau_{0}}=u,\left.\quad I^{-}\right|_{\tau=\tau_{0}}=y .
\end{array}\right.
$$

Here the $v \equiv v(x, y) \equiv v\left(x, y, \tau_{0}\right)$ and $u \equiv u(x, y) \equiv u\left(x, y, \tau_{0}\right)$ are "known" solution of reflection-transmission problem for the same medium (Fig.3). That is, we have an inverse problem of a restoration of internal field intensity by means of known external radiation, given only at the one boundary (left or right) of medium.

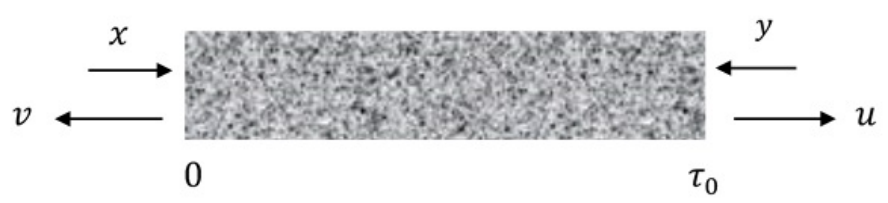

Figure 3: The "known" solution of reflection-transmission problem for the medium $\left[0, \tau_{0}\right]$ 
Direct problem formulation of obtaining the same internal field intensities $I^{+}$and $I^{-}$can be readily constructed by exploring the nonlinear version of the Ambartsumian's "layers addition method" (Ambartsumian 1964a).

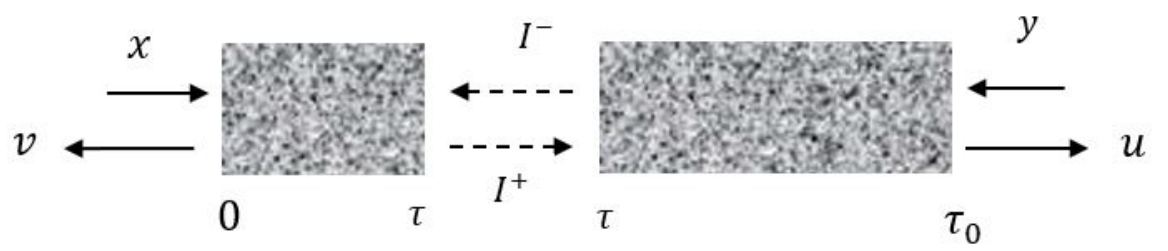

Figure 4: Addition of layers $[0, \tau]$ and $\left[\tau, \tau_{0}\right]$.

If the reflection-transmission properties of two separate layers $[0, \tau]$ and $\left[\tau, \tau_{0}\right]$, respectively, of the finite optical thicknesses $\tau$ and $\left(\tau_{0}-\tau\right)$ are known (Fig.4), than the solution for the composite medium $\left[0, \tau_{0}\right]$ problem can be recast (Pikichyan 2014)] in the form:

$$
\left\{\begin{array}{c}
I^{+}=u\left(x, I^{-}, \tau\right) \\
I^{-}=v\left(I^{+}, y, \tau_{0}-\tau\right)
\end{array} .\right.
$$

Hence, it is obvious that for a determination of the internal field $I^{ \pm}$of radiation in quest, by means of both inverse or direct methods, we need to obtain in advance a solution of reflection-transmission problem $u$ (or/and $v)$.

\section{Solution of reflection-transmission problem}

Non-linear form of Ambartsumian's principle of invariance (Ambartsumian 1964a) (see e.g. . Bellman, Kalaba, Wing 1960) give us two differential equations of invariant imbedding for the function $u(y, x)$ (Pikichyan 2010):

$$
\begin{gathered}
\frac{\partial u}{\partial \tau_{0}}=\alpha(u, y) \frac{\partial u}{\partial y}+\alpha(y, u), \\
\frac{\partial u}{\partial \tau_{0}}=\alpha(v, x) \frac{\partial u}{\partial x}, \quad v(x, y) \equiv u(y, x) .
\end{gathered}
$$

Eliminating the terms which include differentiation over the layer thickness, we obtain the functional equation of Ambartsumian's complete invariance:

$$
\alpha(v, x) \frac{\partial u}{\partial x}-\alpha(u, y) \frac{\partial u}{\partial y}=\alpha(y, u) .
$$

In the linear case,

$$
\alpha(x, y)=\frac{\lambda}{2} x-\left(1-\frac{\lambda}{2}\right) y
$$


and the equation (8) becomes the Ambartsumian's known relation for coefficients of reflection and transmission of finite medium (Ambartsumian 1944):

$$
R^{2}-2 \frac{2-\lambda}{\lambda} R+1=T^{2}
$$

To solve the equation (8), we may use the method of, so-called, "linear images" (Pikichyan 2016, 2014). The solution $u\left(x, y, \tau_{0}\right)$ is found as a linear combination of the two auxiliary functions $-T \equiv T(x, y) \equiv T\left(x, y, \tau_{0}\right)$ and $R \equiv R(x, y) \equiv R\left(x, y, \tau_{0}\right)$. They are called the "linear images" of required solution $u\left(x, y, \tau_{0}\right)$ :

$$
u=T \cdot x+R \cdot y .
$$

In the linear case, the quantities $T$ and $R$ are constants, and they are identical to real reflection and transmission coefficients of medium, illuminated only from one side. In a conservative case, we have $\lambda=1$ and $R+T=1$. We can easily derive, from relations (8) and (9), a symmetrical functional equation for determining the linear image $T$ in quest:

$$
\left[k(x+v) \frac{\partial}{\partial x}+k(y+u) \frac{\partial}{\partial y}\right] T=-T \cdot \frac{k(x+v)-k(y+u)}{x-y},
$$

where $k(\xi)=\frac{k_{0}(l)}{1+b \xi}$. Integration of (10) shows that, in the case at hand, the linear image $T$ depends only on the sum of its arguments $T(x, y) \equiv$ $T(x+y)=T(\xi)$. It can be written explicitly as

$$
T(\xi)=q \frac{1+b \xi}{1+q b \xi}=\frac{2(1+b \xi)}{\tau_{0}+2(1+b \xi)} .
$$

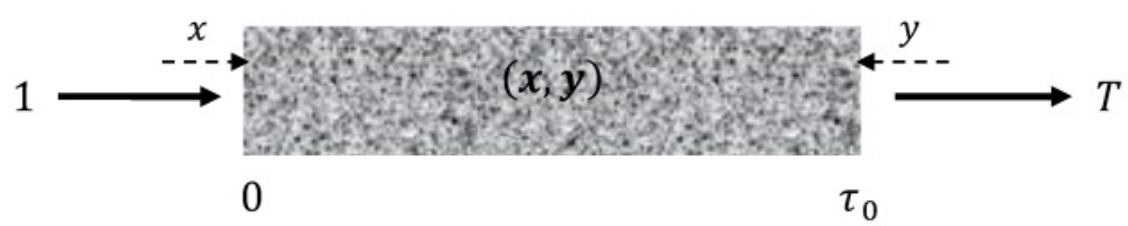

Figure 5: Physical meaning of Linear Image $T\left(x, y, \tau_{0}\right)$

The linear image $T\left(\xi, \tau_{0}\right)$ has a clear physical mining (Fig.5), namely it is the light transmission probability of the layer which has the "limiting" optical depth $\tau_{0}$ (be relative to linear case, i.e. non-exited medium), whereas a single photon or unit beam of radiation falls on to one boundary of medium, but layer is continuously under the bilateral influence of external incident intensities $(x, y)$, respectively. Solution of nonlinear reflection-transmission problem can be written in terms of the linear image as

$$
u(x, y)=y+(x-y) \cdot T(x+y),
$$


note that $v(x, y)=u(y, x)$. So we see that the function $u(x, y)$ (or $v(x, y))$, which depends on two energy variables, can be expressed through the new axillary function $T(\xi)$ of only one variable $\xi \equiv x+y$.

\section{Internal field solution as an inverse problem}

Using the forms (11) and (12), we see that the solutions of both Cauchy problems (4) give us the same result (see also Pikichyan 2018)

$$
I^{+}=I^{-}+(x-y) \cdot T(x+y), \quad A \cdot\left(I^{-}\right)^{2}+B \cdot I^{-}-C=0,
$$

where the coefficients and free member of the quadratic equation set forms $\left(\xi^{ \pm} \equiv x \pm y\right):$

$$
\begin{aligned}
& A=b\left[\tau_{0}+2\left(1+b \xi^{+}\right)\right], \quad B=\tau_{0}+2\left(1+b \xi^{+}\right)\left(1+b \xi^{-}\right), \\
& C=\tau_{0}(1+b x) x-\tau\left(1+b \xi^{+}\right) \xi^{-}+2 y(1+b x)\left(1+b \xi^{+}\right) .
\end{aligned}
$$

Physical solution of the quadratic equation corresponds to a positive sign of the discriminant of the quadratic Trinomial in (13), because it satisfies the linear limit of the problem. Linear task formally corresponds to $b \equiv 0$.

Thus, the functional equation of "Ambartsumian's complete invariance" (8) was derived from principle of invariance for determination of outgoing radiation $u\left(x, y, \tau_{0}\right)$. The last one is then transformed to $(10)$ for $\operatorname{LI} T\left(\xi, \tau_{0}\right)$, which is solved analytically (11). Solution of "reflection-transmission" problem in explicit elementary form (12) was obtained through the LI. Presence of this solution is now a sufficient condition to formulate the Cauchy problem (4), which can be solved analytically in order to "restore" a radiation field inside the medium (13). Thus, the sequence for final determination of unknown quantities is $(\xi \equiv x+y)$

$$
T\left(\xi, \tau_{0}\right) \rightarrow u\left(x, y, \tau_{0}\right) \rightarrow I^{ \pm}\left(\tau, x, y, \tau_{0}\right) .
$$

\section{Internal field solution as a direct problem}

Implications of the concept of LI and formulas (11), (12) in the system (5) for the solution of direct problem give the form

$$
\left\{\begin{array}{c}
\left(1+q_{\tau} b x\right)\left(I^{+}-I^{-}\right)+\left(I^{-} \cdot I^{+}\right) q_{\tau} b=-q_{\tau} I^{-}+(1+b x) q_{\tau} x \\
\left(1+q_{\tau_{0}-\tau} b y\right)\left(I^{+}-I^{-}\right)-\left(I^{+} \cdot I^{-}\right) q_{\tau_{0}-\tau} b=q_{\tau_{0}-\tau} I^{+}-(1+b y) q_{\tau_{0}-\tau} y
\end{array},\right.
$$

where the value $q_{\beta}=2 /(\beta+2)$ is the solution of the linear transmission problem for the layer $[0, \beta]$. Solution of this algebraic system leads to the same expressions (13), which we have already obtained in the inverse problem. 


\section{Plot illustrations}

Below we bring three diagrams of diffuse radiation field intensity dependence of increase of intensity of external radiation (in all calculations conventionally $b \equiv 1)$. Fig. 6 shows the change of the unit beam intensity which is passing through the medium of given optical thickness, while the total exciting power of external radiation increases. Here the initial value $(\xi=0)$ of transmitted radiation corresponds to the linear

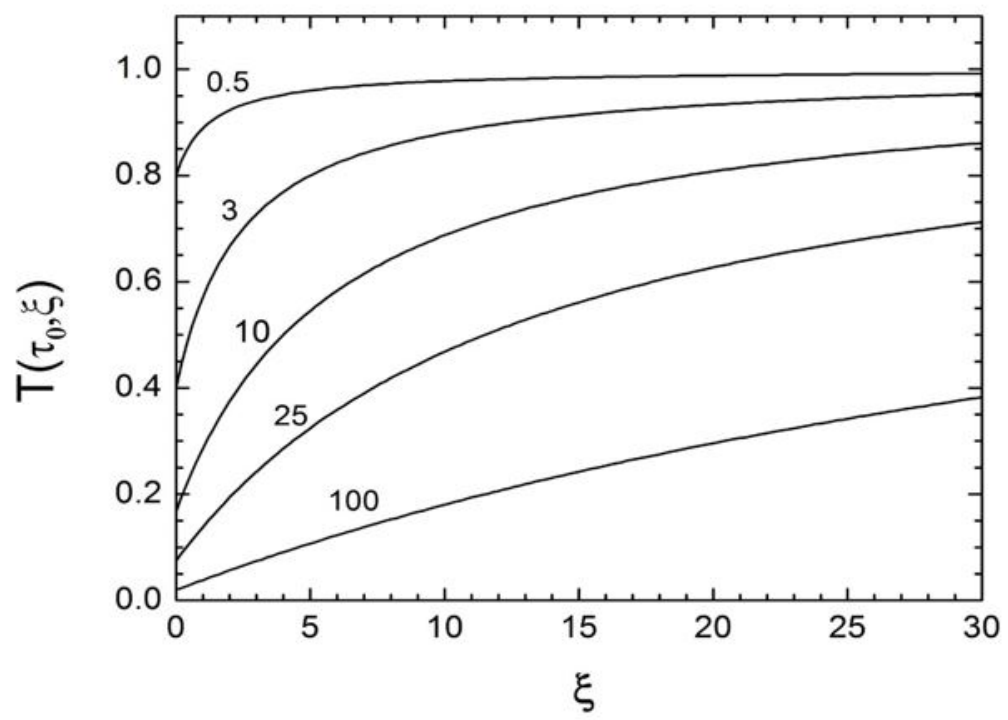

Figure 6: Evolution of medium transmittance $T\left(\xi, \tau_{0}\right)$.

case, i. e. it refers to an unexcited medium. During growth of excitation level $(\xi \rightarrow \infty)$, the transmittance of medium increases (Ambartsumian 1964b) and it becomes more enlightened up to achieve the level of full transparency

$$
\lim _{\xi \rightarrow \infty} T\left(\xi, \tau_{0}\right) \rightarrow 1
$$

Fig. 7 shows the dependence of the transmitted radiation $u\left(x, y, \tau_{0}\right)$ of growth of right-hand external irradiation $y$, while $x=2, \tau_{0}=1$ and $\tau_{0}=10$

The solid curves correspond to nonlinear case, and the dotted curves represent the solutions of linear problem. In comparison with linear case, an increase of radiation field in nonlinear theory is more slowly, because of medium enlightenment phenomena (Ambartsumian 1964b). The deviation between the curves of linear and non-linear tasks continuously increases.

The same, but on a larger scale we see on curves of internal field problem. The Fig. 8 shows the progress of the internal field intensity $I^{ \pm}\left(\tau, x, y, \tau_{0}\right)$ in dependence on growth of external radiation, with $\tau=0.5$ and $\tau_{0}=1$, while 


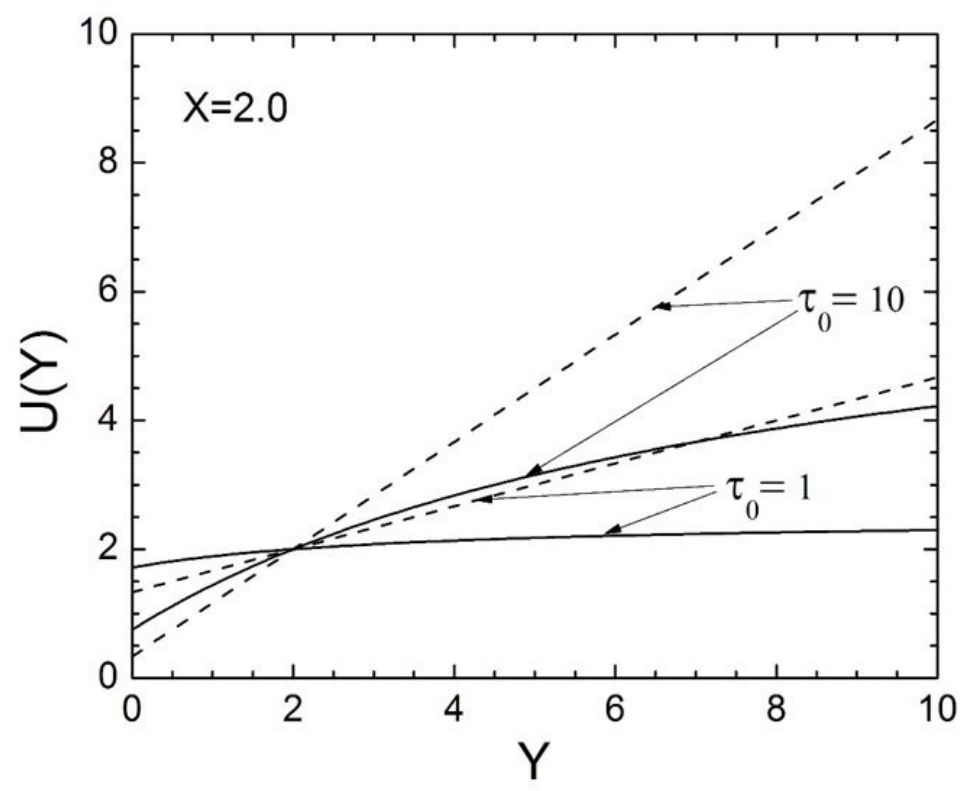

Figure 7: The progress of transmitted radiation $u\left(x, y, \tau_{0}\right)$

$x=y$ (i.e. in the middle of medium of unit optical thickness), when both external boundaries of the slab irradiated with the same value of beams.

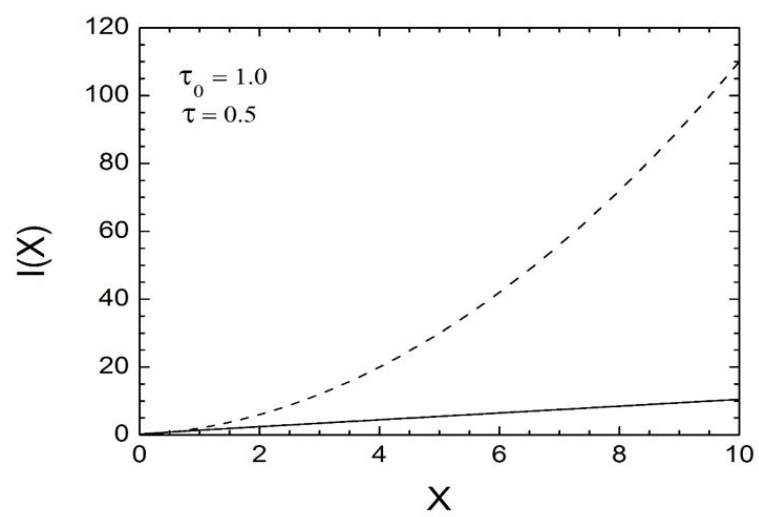

Figure 8: Behavior of internal radiation field $I^{ \pm}\left(\tau, x, y, \tau_{0}\right)$.

\section{Concluding remarks}

In this report, we further expose on the simple example the assertions made in non-linear problem:

1) A definition of linear structures in the solution of nonlinear problem 
of radiative transfer by means of a concept of "linear images" presents a good opportunity for simplification of the solutions of the problems of inverse or direct formulations. In some simple cases, the solution may brought up to an explicit analytic form.

2) When analyzing the fields of diffuse radiation only by means of linear problems of multiple scattering, there is a deficiency of over-estimate of values of real fields of the diffuse radiation, as well as the optical parameters of the medium, even in case of relatively small values of external excitation fields.

\section{References}

Ambartsumian V. A. 1944, Trans. Armenian Acad. Sci. (Izv. Acad. Nauk Arm. SSR, estestv. nauki), №1-2, 31-36, (in Russian).

Ambartsumian V.A. 1964a, Proc. Armenian Acad. Sci. (Dokl. Acad. Nauk Arm. SSR), 38, №4, 225-230, (in Russian).

Ambartsumian V. A. 1964b, Proc. Armenian Acad. Sci. (Dokl. Acad. Nauk Arm. SSR), 39, №3, 159-165, (in Russian).

Bellman R., Kalaba R., Wing M.1960, Proc. Nat. Acad. Sci. USA, 46, 1646-1649.

Ivanov V. V. 1969, Radiative Transfer and Spectra of Celestial Bodies, Science, M., 472 p. (in Russian).

Pikichyan H. V. 2010, Astrofizika, 53, 285-299, 2010 (in Russian); English edition: Astrophysics, 53, 251-267.

Pikichyan H. V. 2014, pp. 48-49, in: Book of abstracts "5-th RussianArmenian Conference on mathematical physics, complex analysis and related issues" (Sept.28- Oct.3, 2014, Yerevan, Armenia), Publ. NAN RA, Yerevan, 56 p. (in Russian).

Pikichyan H. V. 2016, JQSRT, 183, pp.113-127.

Pikichyan H. V. 2018, pp. 64-67, in.: Thesis book of "7-th RussianArmenian Conference on mathematical physics, complex analysis and related issues" (9-15 Sept. 2018, Yerevan, Armenia), Publ. house "Gituthyun" of NAS RA, Yerevan, 84 p. (in Russian). 